\title{
La escolarización de los saberes: un escenario relevante para rastrear y comprender algunos problemas sociales y bumanos contemporáneos
}

The Schooling of Knowledges: a Significant Stage to Track and Understand some Contemporary Social and Human Problems

La scolarité de savoirs : un scénario important pour suivre la trace et comprendre quelques problèmes sociaux et humains contemporains

A escolarização dos saberes: um palco relevante para rastrear e compreender alguns problemas sociais e humanos contemporâneos

Fecha de recepción: 11 DE NOVIEMBRE DE 2015/Fecha de aceptación: 24 DE JULIO DE 2015/Fecha de disponibilidad en línea: 15 DE MARZO DE 2016

Encuentre este artículo en http://magisinvestigacioneducacion.javeriana.edu.co/

\section{Resumen}

El texto perfila un horizonte de poco interés para los lugares dominantes de producción discursiva sobre educación, currículo y formación. Se trata de algunas tensiones y modificaciones en el sentido del conocimiento que desafían los procesos, agentes e instituciones vinculadas con el proceso de escolarización de los saberes en la actualidad. Entre la apuesta dominante por una valoración instrumental y una opción que reivindica un sentido de las humanidades canónicas eurocéntricas, se avizora una alternativa en la exploración de saberes menospreciados o desconocidos por las miradas que han dominado en la academia y la escuela.

\section{Palabras clave}

Educación básica; formación de docentes; plan de estudios; saberes locales; conocimientos autóctonos

Para citar este artículo / To cite this article / Pour citer cet article / Para citar este artigo

Parra-Mosquera, C. A. (2016). La escolarización de los saberes: un escenario relevante para rastrear y comprender algunos problemas sociales y humanos contemporáneos. magis, Revista Internacional de Investigación en Educación, 8 (17), 179-186. http://dx.doi.org/10.11144/Javeriana.eser 


\section{Key words}

Primary education; teacher development; curriculum; local knowledge; indigenous knowledge

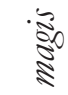

PÁGINA 180

\section{Mots clés}

Education basique; formation des enseignants; plan d'études; savoirs locaux; connaissances autochtones

\section{Palavras-chave}

Educação básica; formação de docentes; plano de estudos (currículo); saberes locais; conhecimentos autóctones

\section{Abstract}

The text outlines a horizon of little interest to the dominant discursive production on education, curriculum and teacher development. These are some tensions and modifications within the meaning of knowledge that currently challenge the processes, actors and institutions involved in the schooling process. Among the dominant view for an instrumental assessment and an option that vindicates the meaning of Eurocentric canonical humanities, an alternative is in sight regarding the exploration of under valued or unknown knowledges by the views that have dominated the academy and the school.

\section{Résumé}

Le texte trace un horizon de peu intérêt visà-vis les lieux dominants de production discursive par rapport à l'éducation, le curriculum et la formation. II s'agit de quelques tensions et modifications dans le sens de la connaissance qui défient les processus, agents et institutions liées au processus de scolarité des savoirs dans l'actualité. Entre I'enjeu dominant d'une estimation instrumentale et le choix qui revendique un sens des humanités canoniques eurocentriques, on vise une alternative dans l'exploration de savoirs méprisés ou inconnus par les regards qui ont étés dominants à l'académie et à l'école.

\section{Resumo}

O texto apresenta um horizonte de pou co interesse para os lugares dominantes de produção discursiva sobre educação, currículo e formação. Trata-se de algumas tensões e modificações no sentido do conhecimento que desafiam os processos, agentes e instituições vinculadas com o processo de escolarização dos saberes na atualidade. Entre a aposta dominante por uma valorização instrumental e uma opção que reivindica um sentido das hu manidades canônicas eurocêntricas, se vislumbra uma alternativa na exploração de saberes menosprezados ou desconhecidos pelos olhares que têm dominado a academia e a escola. 
En tiempos del capitalismo cognitivo están dándose, y aún no terminan de establecerse, modificaciones en el sentido del conocimiento que tienen repercusiones profundas en las concepciones de lo educativo, de la formación de los sujetos, del oficio de maestro y de la escuela. Estas transformaciones no se agotan ni limitan a las tecnologías de la información y la comunicación, TIC, como se cree habitualmente en las definiciones predominantes referidas a la sociedad del conocimiento. El hecho de que los conocimientos más relevantes sean aquellos que pueden ser patentados y que son necesarios para el desarrollo de tareas, para la gestión y la toma estratégica de decisiones, puede rastrearse en diversas regiones de la sociedad en general y en la educación en particular. Precisamente, en el escenario del capitalismo cognitivo se entienden algunos de los mecanismos mediante los que el mercado captura el mundo de la vida. En la transición del fordismo al posfordismo, se pasa de producir productores y vida biológica a producir consumidores y vida como información, y emerge una economía fundamentada en la difusión del saber, en el que la producción de conocimiento juega un rol principal en la valorización del capital. De igual modo, lo que se produce y se vende no son solamente bienes materiales o inmateriales, sino formas de vida, formas de comunicación, patrones de socialización, de educación y de percepción (Castro-Gómez, 2009; Galcerán, 2007).

En esta perspectiva, trazada escuetamente, es viable examinar algunas ideas orientadas a comprender los cambios en el sentido del conocimiento en un escenario y un proceso concretos: la escolarización de los saberes. La emergencia, la dilución o la modificación de un saber en la escuela involucran las complejas lógicas y procesos históricos y sociales en que están inmersos los campos académicos y de las políticas educativas, y vinculan las dinámicas y prácticas cotidianas de la escuela y la sociedad. Por ello, antes que ser un proceso neutral, la incursión, la desaparición o la modificación de un saber en la escuela visibilizan y acentúan unos sentidos y desestiman o invisibilizan otros, porque tienen efectos en los sentidos, los contenidos y las maneras de entender cómo se conoce, qué es conocimiento, cómo este se materializa y divulga, cómo se nombra, para qué y para quién se conoce.

Al respecto, vale la pena presentar tres ejemplos que pueden leerse como signos de la hegemonía del mercado en la educación y, quizá, del fascismo societario planteado por Boaventura de Sousa-Santos, cuando advierte:

Si se permite que la lógica del mercado se desparrame de la economía a todos los campos de la vida social y se convierta en el único criterio para establecer interacciones sociales y políticas, la sociedad se tornará ingobernable y éticamente repugnante (2009, p. 227).

En primer lugar, desde la relación TIC-educación, se abrió la puerta con fuerza a la presencia del sector productivo en los años noventa', ya no solo como referente para la educación colombiana, "con miras a responder a la apertura de mercados, al reto del desarrollo tecnológico y a las exigencias de un desarrollo humano y social equilibrado" (Bernal-Ramos, 1992, p. 195), sino también como agente y participante de la reconversión del sistema educativo y del sentido de lo educativo, por medio de producciones investigativas y programas de formación de maestros, como los
Descripción del artículo | Article description | Description de l'article | Artigo descrição

Este ensayo examina, desde la experiencia investigativa del autor, algunas ideas orientadas a comprender los cambios en el sentido del conocimiento en el marco de la escolarización de los saberes. Este proceso se perfila como un escenario plausible para explorar alternativas frente a transformaciones sociales contemporáneas de gran envergadura en el ámbito de las subjetividades, las instituciones y las relaciones de saber-poder en tiempos del capitalismo cognitivo.

1 Entendido como la empresa privada y luego, más específicamente, la relacionada con las telecomunicaciones. 
de Intel, de la Fundación Telefónica y de Microsoft. En segundo lugar, sirve referir el programa de Educación Financiera de la Fundación Bancolombia para mostrar que los cambios en el sentido del conocimiento, en cuanto a la escolarización de los saberes, no se agotan en las TIC ni se deben a estas únicamente. Se trata de un programa que "busca el desarrollo de competencias financieras para hacer un mejor uso de los recursos económicos disponibles, tomar decisiones propias y ser socialmente responsables. Para la aplicación del proyecto del Área de Responsabilidad Social de Bancolombia y de la empresa Qualificar, capacitarán a 2.080 docentes del país en temas como antecedentes del proceso financiero y estrategias pedagógicas sobre la economía, entre otras" (El Tiempo, 1 de marzo de 2010). En tercer lugar, vale remitir a las políticas educativas como mecanismo y ámbito fértil en la manera en que el mercado ha ido capturando la educación. Muestra de ello han sido las reformas educativas de grandes dimensiones en el campo de las políticas educativas que inician en la década de los noventa en el país y en América Latina. Estas reformas han establecido, fundamentalmente, nuevos parámetros en la relación público-privado, pasando del derecho de la educación al servicio educativo, y han afianzado el discurso y la lógica de la gestión empresarial y estratégica para las orientaciones y decisiones académicas e institucionales (Martínez-Boom, 2004).

Las maneras en que los saberes se escolarizan dejan leer los intereses y exigencias de la sociedad y del Estado sobre la escuela y la formación de los sujetos, y también permiten documentar los signos de las transformaciones en los sentidos del conocimiento $y$, por qué no, ofrecen herramientas para arriesgar miradas alternativas. Actualmente, se reconoce el fortalecimiento de una mirada sobre el mundo, el conocimiento y los sujetos, en la que, a modo de interrogante y alerta, se avizora el predominio de la razón instrumental, anunciando las connotaciones profundas de convertir o intentar convertir todo en materia prima, en un hacer, en una aplicación rentable y tangible. Desde finales del siglo XIX, en la escuela y en los fines educativos que se le exigían a esta desde el Estado y la sociedad, empezaba a privilegiarse el sentido del conocimiento hacia la aplicación, la acción, la eficacia y la productividad, en desmedro de otros sentidos formativos y valores del conocimiento, como los asociados a las artes y las humanidades.

La complejidad que en la sociedad moderna asume la división social del trabajo impone una mirada analítica y un criterio cada vez más pragmático. Al respecto, dice John Franklin Bobbitt:

Deben evitarse aquellos objetivos que no son sino aspiraciones y esperanzas vagas o rimbombantes.
Ejemplos de esto son la formación del carácter, el desarrollo armónico del individuo, la eficiencia social, la disciplina general, la cultura, etc. Todos estos siguen siendo válidos, pero son demasiado nebulosos para servir de guías en la práctica. Pertenecen a la visionaria adolescencia de nuestra profesión; no a la prudente y un tanto desengañada madurez (Bobbitt, 1924, citado por Martínez-Boom, Noguera \& Castro, 2003, p. 75).

En este sentido, la tensión entre los fines educativos nebulosos y los que no lo son inspiró y llevó a la educación a centrarse en:

[...] el diseño y programación minuciosa de las diferentes tareas que debía ejecutar cada obrero en su puesto de trabajo, así como de los instrumentos y herramientas que debería utilizar para realizar más efectivamente su labor. El job analysis y su instrumento, el task analysis, constituyen, pues, la base misma de la teoría curricular de comienzos de siglo (Martínez-Boom, Noguera \& Castro, 2003, p. 75).

Al parecer, antes del advenimiento de la informática y de la automatización de procesos en la posguerra, ya se habían trasladado formas, sentidos y agentes a la educación y a la escuela afines a la preocupación de la fábrica. Pero la referencia al job analysis y al diseño instruccional en el giro del sentido del conocimiento no termina allí. La tradición anglosajona sobre el currículo (a la cual pertenece Bobbitt) también es la que ha fundamentado con fuerza la tecnología instruccional y la tecnología educativa que se han apropiado en el campo de las políticas educativas en Colombia. En efecto, el dilema entre los objetivos educativos nebulosos y los que no lo son se resolvió en favor de los segundos, es decir, de aquellos objetivos que servían de "guías en la práctica", decisión esencial para fundamentar el trabajo del Servicio Nacional de Aprendizaje, SENA, en Colombia desde 1957 y de la tecnología educativa en la mirada dominante sobre el currículo y la educación. Este dilema se desplazó con igual resultado, ya no solo para pensar la formación de los estudiantes, sino también de los maestros, en términos de competencias profesionales.

Ahora bien, en el ámbito nacional e internacional, la evaluación y el currículo han configurado una alianza estratégica cimentada en complejos y profundos procesos históricos que, día a día, ganan fuerza (verdad) y sofisticación en las reacomodaciones del discurso sobre la educación y en sus concreciones y efectos cotidianos (dominaciones y resistencias) en la escuela y en las instituciones formadoras de maestros (Martínez-Boom, 2004). Precisamente, el concepto de competencia es una bisagra y piedra angular en los 
complejos efectos de la alianza entre evaluación y currículo, en la cual, sin duda, los conceptos y sus operacionalizaciones en diversos ámbitos (financiación de proyectos, investigación educativa, eventos académicos, programas de formación, actividades escolares) juegan un papel estratégico. A modo de ejemplo, los Estándares Básicos de Competencias "constituyen los parámetros de lo que todo niño, niña y joven debe saber y saber hacer para lograr el nivel de calidad esperado a su paso por el sistema educativo y la evaluación externa e interna" (MEN, 2006, p. 9). Estos se traducen, básicamente, en lineamientos nacionales y locales para las regiones, instituciones y profesores en torno al tipo de contenidos, metodología de enseñanza e indicadores de desempeño de los estudiantes en cada una de las áreas del conocimiento. Igualmente, los estándares marcan las pautas en la orientación y las formas de las evaluaciones masivas que examinan a los estudiantes, pero también a los profesores e instituciones educativas. Un mal desempeño en las pruebas SABER afecta la financiación de las instituciones —oficiales y no oficiales_-, por ende, tiene efectos en la cotidianidad escolar y el oficio de maestro (en qué y cómo distribuir el tiempo y el espacio escolar, por ejemplo). Siendo bastante osados, en el caso del área del lenguaje, vale preguntar cuáles serían los maestros productivos y no productivos. Sin ser maniquea o reduccionista frente a la complejidad de los dos sentidos, ¿cuál de los dos calificativos utilizaríamos para los maestros que prefieren leer literatura en voz alta a niños de quinto de primaria en lugar de entrenarlos para las pruebas SABER de lenguaje?, ¿cómo los verán los colegas, los padres, los estudiantes, cómo se verán ellos mismos?, ¿cuál de las dos prácticas arrojaría más evidencias o indicadores tangibles?

Adicionalmente, si revisamos someramente las seis grandes metas de la formación en lenguaje en la educación básica y media, a saber: la comunicación; la transmisión de información; la representación de la realidad; la expresión de los sentimientos y las potencialidades estéticas; el ejercicio de una ciudadanía responsable y el sentido de la propia existencia (MEN, 2006), podríamos preguntar ¿cuáles de estas áreas permitirían puntuar mejor en las pruebas?, ¿a cuáles se les podría establecer indicadores de desempeño observables y confiables? Y, por extensión, ¿cuáles metas atraparían la atención e interés en la cotidianidad escolar y cuáles se diluirían? Al parecer, las metas de comunicación y transmisión de información son proclives a la elaboración de indicadores: en un texto escrito, por ejemplo, se puede crear una escala evaluativa que indague por el uso de conectores (cantidad y variedad) y que permita ordenar el desempeño de los estudiantes antes y después de un período escolar. Pero esta tarea se dificulta o se torna absurda cuando se intenta vincular la lógica de las competencias y de los indicadores observables y tangibles a metas formativas "nebulosas" como la expresión de los sentimientos y las potencialidades estéticas; el sentido de la propia existencia o el ejercicio de una ciudadanía responsable, sin incurrir en el artificio o la instrumentalización de la autonomía, el goce estético o el diálogo intercultural.

De otro lado, las ciencias exactas se han ponderado prioritariamente por su utilidad y productividad para el desarrollo individual y social en relación con las TIC en Colombia, más específicamente con la informática, en una lógica en donde "la rápida obsolescencia del conocimiento y de la tecnología" (Llinás, Aldana, Chaparro, Gutiérrez, Palacios, Patarroyo, Posada, Restrepo, Vasco \& García-Márquez, 1995, p. 80) ayuda a explicar la educación permanente y diversos programas multimillonarios en dotación de equipos y capacitación para su manipulación. Así, ha sobresalido una mirada de la ciencia y del conocimiento reduccionista porque se afinca en el discurso del sector productivo (calidad, innovación, investigación y 
desarrollo) y está escindida de otras áreas del saber y aspectos sociales. Esta lógica materializada y agenciada de diversas maneras desde hace décadas, también lleva a pensar en otras definiciones o sentidos de conocimiento que se excluyen o menosprecian en la escuela y ven como inútiles o dejan por fuera otros saberes y formas de conocimiento: ¿rápida obsolescencia del arte, de la literatura, de la historia?

La superioridad de algunos sistemas educativos, en especial la alfabetización computacional, el entrenamiento en ciencias básicas, matemáticas, química, física y la experiencia en laboratorios, así como el fomento del talento para la innovación, generan un mejor nivel de competitividad para los futuros profesionales en ciencia e ingeniería (Llinás, Aldana, Chaparro, Gutiérrez, Palacios, Patarroyo, Posada, Restrepo, Vasco \& García-Márquez, 1995, p. 33)

Ahora bien, Martha Nussbaum (2010) ha leído la incursión del mercado en los saberes escolarizados como una expresión de la crisis educativa y social. Ella plantea que:

se están descartando sin advertirlo ciertas aptitudes que son necesarias para mantener viva a la democracia [...] se están erradicando materias y carreras relacionadas con las artes y las humanidades (en todos los niveles educativos) en un momento en que las naciones deben eliminar todo lo que no tenga ninguna utilidad para ser competitivas en el mercado global (p. 20) La idea de la rentabilidad convence a numerosos dirigentes de que la ciencia y la tecnología son fundamentales para las naciones en el futuro (p. 25).

Sin embargo, en la apuesta de esta autora, se lee un acento reivindicativo del arte y las humanidades canónicas, herederas de concepciones segregacionistas entre alta y baja cultura y de su división con las ciencias y la filosofía, así como una lectura monolítica de las TIC y las ciencias naturales como agente exclusivamente instrumentalizador. Interesa insistir en este reparo porque al reforzar una división entre ciencias sociales y humanas y ciencias naturales en el sentido del conocimiento, se restringe la posibilidad de elaborar propuestas y de leer los movimientos que sugieren la emergencia (consolidación) de una mirada sobre el conocimiento, el mundo y los sujetos en la que se anuncia la refinación de la hegemonía del mercado y de la razón instrumental para subordinar o desplazar otros propósitos, prioridades, posibilidades y circunstancias, también relevantes, en el ámbito de la formación de los sujetos. En efecto,

Intuitivamente puede reconocerse, sin dificultad alguna, que en el proceso de generación de conocimientos novedosos la imaginación desempeña un papel determinante. La dificultad se encuentra en otros lugares: por ejemplo, en el reconocimiento social e institucional de los juegos y los ejercicios imaginativos [...] La racionalidad de tipo económico suele ser la principal dificultad en este plano. No sin razón, sugerencias en esta dirección rayan con la utopía (Maldonado, 2005, p. 121)

Ante este panorama, parece viable plantear que, a la hegemonía global del mercado en la sociedad debe planteársele una contrahegemonía que, en general y según Boaventura de Sousa-Santos, requiere un viraje a lo local. Esto no significa una apología nostálgica del ostracismo ni del localismo, sino que está más próximo a una sociología de las ausencias, que 
buscaría inventar o develar "cualquier condición, experimento, iniciativa o concepción política y social suprimida con éxito por las formas hegemónicas de la globalización, o aquellas que no se ha permitido que existan ni sean pronunciables como necesidad o aspiración" (2009, p. 233). En este sentido, es plausible considerar que, en el caso de la educación, esta contrahegemonía podría intentarse mediante la incorporación de saberes inéditos en la escuela y en la formación de maestros, en aras de apostarle a una concreción de la justicia cognitiva, que el mismo De Sousa-Santos define como condición para la justicia social. Inmersión y experimentación con saberes no reconocidos oficial ni institucionalmente en relación con la corporeidad, las emociones, lo indígena, lo afro, la ecología y las poéticas de las TIC, por ejemplo. Estos saberes han sido desdeñados o desconocidos por las miradas dominantes en la academia y en la escuela. Saberes no rentables, pero sí indispensables "donde se dirimen el sentido y el futuro de nuestras escuelas y universidades" (Martín-Barbero, 2009, p. 23).

Ahora bien, no basta con la simple incorporación de nuevos saberes en forma de adición de contenidos o temas, porque así serían fácilmente despojados de sus lógicas particulares. No se ajustarían a las miradas clásicas sobre el conocimiento que se han promovido en Occidente mayoritariamente $y$, por ende, en la escuela. Estas miradas han sido fundadas básicamente en el antropocentrismo y la escisión entre ciencias sociales, humanas y naturales; en el determinismo, el relativismo, el universalismo o el particularismo. Allí ha sobresalido una:

visión del universo como un sistema mecánico compuesto de piezas, la del cuerpo humano como una máquina, la de la vida en sociedad como una lucha competitiva por la existencia, la creencia en el progreso material ilimitado a través del crecimiento económico y tecnológico y, no menos importante, la convicción de que una sociedad en la que la mujer está por doquier sometida al hombre, no hace sino seguir las leyes naturales (Capra, 2003, p. 28).

Otros saberes y sus lógicas no clásicas aportarían y exigirían la apertura a nuevas miradas del mundo, asunto que es consistente con las supuestas tareas del ámbito educativo y formativo. De igual modo, un escenario posible para tal fin sería el de la escolarización de nuevos saberes y, por qué no, el de la experimentación y resignificación de los saberes vigentes actualmente en la escuela. Este proceso interpelaría la lógica educativa tradicional, pues se vincularía con disposiciones éticas y tensiones de saber-poder relacionadas con la inclusión de las lógicas particulares de esos nuevos saberes no clásicos en la escuela clásica. Estos requieren una incorporación que no replique las asimetrías y divisiones entre las áreas del conocimiento y de estas con la naturaleza y la realidad. Igualmente, resultaría relevante explorar los aportes e inquietudes asociados a las ciencias de la vida, con miras a trazar alternativas distintas a la crítica o a la sempiterna elaboración de diagnósticos en el campo educativo y pedagógico. Vale recordar que un "cambio de paradigmas requiere una expansión no solo de nuestras percepciones y modos de pensar, sino también de nuestros valores" (p. 31)

Hasta ahora, estas reflexiones logran, por lo menos, insinuar que la escolarización de los saberes es un escenario plausible para comprender transformaciones sociales contemporáneas de gran envergadura en el ámbito de las subjetividades, las instituciones y las relaciones de saberpoder. Un escenario de posibilidades para encontrar y fortalecer miradas alternativas (en cuanto a otras preguntas, referentes y métodos) para com- 
prender retos y encrucijadas de la escuela y la sociedad contemporáneas, con el fin de aportar en el trazo de un mapa en el cual "encontrar puntos de referencia, que nos permitan situarnos y asumir posición no únicamente desde la unidireccional perspectiva del mapa educativo diseñado por las agencias educativas, por la normatividad, por las lógicas del poder" (MartínezBoom, 2004, p. 410).

\section{Sobre el autor}

Carlos Andrés Parra-Mosquera es docente universitario, licenciado en educación básica con énfasis en humanidades, lengua castellana, magíster en educación, Universidad de Antioquia. Candidato a doctor en ciencias sociales y humanas, Pontificia Universidad Javeriana. Integrante del grupo de investigación Somos Palabra: Formación y Contextos.

\section{Referencias}

Bernal-Ramos, L. (1992). Tecnología y educación: mitos, ritos y retos. Revista Informática Educativa, 5 (3), 183-197. Disponible en: http://rie.uniandes. edu.co/Volumen5.aspx

Capra, F. (2003). La trama de la vida. Una nueva perspectiva de los sistemas vivos. Barcelona: Anagrama.

Castro-Gómez, S. (2009). Noopolítica y sociedades de control: las subjetividades contemporáneas en Mauricio Lazzarato. En Jorge Eliécer MartínezPosada \& Fabio Orlando Neira-Sánchez (comps.) Miradas sobre la subjetividad, 21-38. Bogotá: Universidad de La Salle. Disponible en: http:// myslide.es/documents/libro-miradas-sobre-lasubjetividad.html

El Tiempo (1 de marzo de 2010). Clases de finanzas para 130 colegios públicos. Disponible en: http:// www.eltiempo.com/archivo/documento/MAM3861919

Galcerán, M. (2007). Reflexiones sobre la reforma de la universidad en el capitalismo cognitivo. Nómadas, 27, 86-97. Disponible en: http://www. redalyc.org/articulo.oa?id $=105116595008$

Llinás, R.; Aldana, E.; Chaparro, L. F.; Gutiérrez, R.; Palacios, M.; Patarroyo, M. E.; Posada, E.; Restrepo,
Á.; Vasco, C. E. \& García-Márquez, G. (1995). Colombia, al filo de la oportunidad. Informe conjunto. Misión de Ciencia, Educación y Desarrollo. Bogotá: Magisterio. Disponible en: http:// repositorio.colciencias.gov.co/handle/11146/265

Maldonado, C. (2005). Heurística y producción de conocimiento en la perspectiva CTS. En Iliana Hernández (comp.). Estética, ciencia y tecnología: creaciones electrónicas y numéricas, 98-127. Bogotá: Pontificia Universidad Javeriana.

Martín-Barbero, J. (2009). Colombia: una agenda de país en comunicación. En Jesús Martín-Barbero (coord.). Entre saberes desechables, y saberes indispensables. Agendas de país desde la comunicación, 11-36. Bogotá: Centro de Competencia en Comunicación, C3. Disponible en: http://myslide.es/documents/entre-saberesdesechables-y-saberes-indispensables.html

Martínez-Boom, A. (2004). De la escuela expansiva a la escuela competitiva: dos modos de modernización en América Latina. Bogotá: Anthropos, Convenio Andrés Bello.

Martínez-Boom, A.; Noguera, C. \& Castro, J. (2003). Currículo y modernización. Cuatro décadas de educación en Colombia. Bogotá: Cooperativa Editorial Magisterio, Universidad Pedagógica Nacional.

Ministerio de Educación Nacional, MEN (2006). Estándares básicos de competencias en lenguaje. Bogotá: Ministerio de Educación Nacional, MEN. Disponible en: http://www.mineducacion.gov. co/cvn/1665/article-116042.html

Nussbaum, M. (2010). Sin fines de lucro. Por qué la democracia necesita de las humanidades. Buenos Aires: Katz Editores.

Parra-Mosquera, C. A. (2012). TIC, conocimiento, educación y competencias tecnológicas en la formación de maestros. Nómadas, 36, 145159. Disponible en: http://www.redalyc.org/ pdf/1051/105124264010.pdf

Sousa-Santos, Boaventura de (2009). Una epistemología del sur. La reinvención del conocimiento y la emancipación social. Buenos Aires: Consejo Latinoamericano de Ciencias Sociales, CLACSO, Siglo XXI. 